\title{
Smart EVs Charging Scheme for Load Leveling Considering ToU Price and Actual Data
}

\author{
Jun-Hyeok Kim* and Chul-Hwan Kim ${ }^{\dagger}$
}

\begin{abstract}
With the current global need for eco-friendly energies, the large scale use of Electric Vehicles (EVs) is predicted. However, the need to frequently charge EVs to an electrical power system involves risks such as rapid increase of demand power. Therefore, in this paper, we propose a practical smart EV charging scheme considering a Time-of-Use (ToU) price to prevent the rapid increase of demand power and provide load leveling function. For a more practical analysis, we conduct simulations based on the actual distribution system and driving patterns in the Republic of Korea. Results show that the proposed method provides a proper load leveling function while preventing a rapid increase of demand power of the system.
\end{abstract}

Keywords:Electric vehicle, Charging load, Time-of-Use price, Smart charging, Load leveling

\section{Introduction}

These days sourcing and developing eco-friendly energies is a prominent research area. In addition, many regulations to protect the environment and humans from pollution have been established. The Kyoto protocol and Copenhagen climate change conferences have significantly contributed to the establishment of these regulations. With this trend, the interest in EVs has consequently increased, as they neither consume fossil fuel nor emit harmful gases [1]. However, since EVs use electricity for their power source, they need to be charged from an electrical power system. When large-scale EVs are being charged simultaneously, various severe phenomena affecting the power system could occur. In [2-10], the possible effects on the power system are described, including voltage variation, decrease of load factor, increase of power losses, increase of demand power, etc. If compensation devices are used to mitigate these negative effects, additional costs would be incurred. Thus, many studies focus on the development of an optimal EV charging scheme to minimize or mitigate any adverse effects due to charging of EVs. EVs can have a frequency regulation function for charging or discharging [2-3]. It has also been shown that EVs can be used for voltage regulation [4]. In [9-10], it was shown that the way EVs are charged can mitigate the intermittence of the renewable energy output. Some papers propose charging schemes for EVs to mitigate the possible negative effects. In [11], an optimal EV charging scheme is proposed to reduce voltage variations and power losses. This charging scheme computes an optimal charging profile of EVs by minimizing the power losses with quadratic programming.

$\dagger$ Corresponding Author: Dept. of Electrical and Electronic Engineering, Sungkyunkwan University, Korea. (chkim@skku.edu)

* Dept. of Electrical and Electronic Engineering, Sungkyunkwan University, Korea. (kjh30309@skku.edu)

Received: February 26, 2016; Accepted: May 31, 2016
In [12], a method was proposed that can reduce the voltage variations and power losses and thus maximize the load factor. The study explored the relationship between feeder losses, load factor, and load variance. An optimal charging scheme was then developed based on this relationship. In [13], a smart EV charging profile was used to assess the impact of EVs on a specific region. However, since the smart charging profiles used were fixed, this method is only acceptable under specific conditions. An optimal EV charging scheme was proposed in [14] that can provide a valley filling function. The decentralized protocol, proposed in the paper, has an advantage when the amount of data increases due to the large scale of EVs. In [15], an EV charging scheme for valley filling was also suggested. However, it is assumed that all the EVs are connected in ideal conditions such as equal capacity and equal required time for charging, and thus is not sufficiently practical. All of the proposals summarized above have the common limitation of not considering the EV owners' driving patterns. The initial State-of-Charge (SoC) of each EV, when it is connected to the power system, is directly related to the driving distance of the EV. Therefore, consideration of the computation of the initial SoC by using a stochastic driving distance is one of the most important factors for practical analysis.

The contributions of this paper include the following.

(1) We consider the EV owner's driving pattern; As mentioned above, this is one of the most important factors for practical analysis of the possible effect of EVs on the power system.

(2) We consider the EV owners' responses to the EV charging scheme; Most papers that propose a smart charging scheme for EVs only consider the Power System Operator (PSO). That is, it is assumed that EV owners would follow any proposed control scheme, which is unrealistic. Thus, the smart EV charging scheme proposed 
in this paper is based on a ToU price based charging scheme that can reflect the response of each EV owner to the proposed charging scheme.

The remainder of this paper is organized as follows. Section II describes a computation of the EV load. In addition, Gaussian distribution based on actual traffic volume is used for the calculation of the initial SoC of each EV. Section III describes the characteristics of several EV charging schemes, including the smart EV charging scheme. Section IV shows comparisons of simulation results depending on the different types of EV charging schemes. The conclusion is then given in section $\mathrm{V}$.

\section{Daily Load and EVs Charging Profile}

In this section, the daily load and EV charging profile are explained. To compute the daily load, we use several actual data from Seoul, Republic of Korea. The estimation of the initial SoC is an important factor in the computation of an EV's charging profile. To calculate the initial SoC, the driving pattern needs to be evaluated, whereby the starting time and duration for charging are the main variables on the EV load. The estimation of the initial SoC can be divided into three main areas as follows. (1) Set the total number of EVs using actual data in Seoul, Republic of Korea, (2) estimate the driving distance of each EV using the actual driving pattern, and (3) calculate the initial SoC of each EV using Gaussian distribution.

\subsection{Daily load profile}

To set the total number of EVs, we selected a specific region in Seoul, Republic of Korea for more practical analysis. The total length of the power system is $1.5 \mathrm{~km}$ and consists of two-step-type poles and a neutral line. Electric power is delivered to 13 loads in the upper portion and 11 loads in the bottom portion. The total active and reactive loads are 28.6 MW and 13.9 Mvar, respectively. In

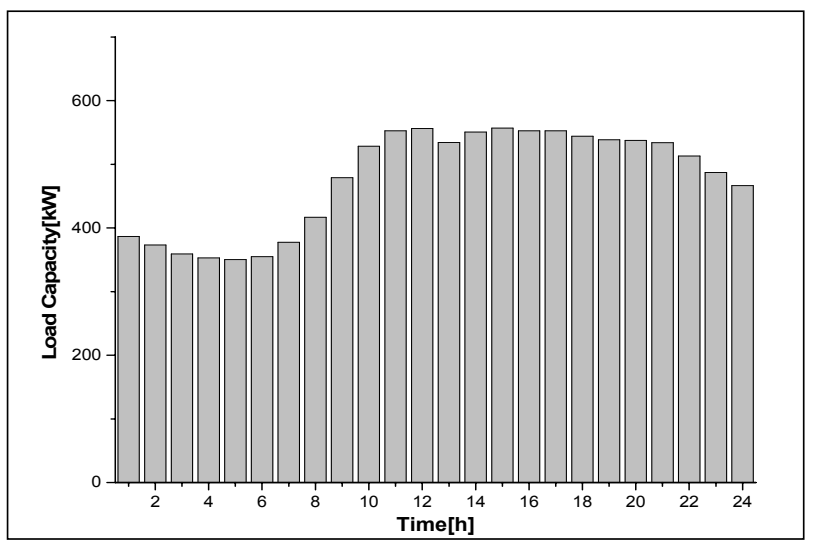

Fig. 1. Daily load profile for specific region in Seoul, Republic of Korea (summer) this paper, we only considered the bottom portion of the power system [16-17]. Fig. 1 shows the daily load profile in the region in which the EV load is not taken into account.

\subsection{Estimation of the number of EVs}

An estimation of the number of EVs is required for the next procedure. According to [18], the actual number of compact vehicles in the region in 2012 was 8,961. Since the Republic of Korea government set a target that by, $10 \%$ of the total number of compact vehicles will be replaced with EVs [19], we assumed that the current total number of EVs in the region is 896. The next step is to determine the number of EVs at each time and load point. To calculate the number of EVs at each load point, we make an

Table 1.The number of EVs at each load point

\begin{tabular}{c|c|c|c}
\hline Location & Load Capacity[kW] & Number of EVs & Load Ratio [\%] \\
\hline L1 & 1189 & 77 & 8.574 \\
\hline L2 & 382 & 25 & 2.754 \\
\hline L3 & 843 & 54 & 6.079 \\
\hline L4 & 1792 & 116 & 12.927 \\
\hline L5 & 565 & 37 & 4.074 \\
\hline L6 & 727 & 47 & 5.243 \\
\hline L7 & 1194 & 77 & 8.610 \\
\hline L8 & 1955 & 77 & 8.618 \\
\hline L9 & 1282 & 83 & 9.245 \\
\hline L10 & 305 & 20 & 2.199 \\
\hline L11 & 4392 & 284 & 31.674 \\
\hline Total & 13866 & 896 & 100 \\
\hline & & &
\end{tabular}

Table 2. The number of vehicles and traffic volume ratio at each hour

\begin{tabular}{|c|c|c|c|c|c|c|}
\hline Time & Commute & Personal & School & Business & Total & $\begin{array}{l}\text { Rate } \\
{[\%]}\end{array}$ \\
\hline 0 & 672 & 437 & 159 & 710 & 1979 & 0.89 \\
\hline 1 & 255 & 172 & 11 & 339 & 776 & 0.35 \\
\hline 2 & 135 & 63 & 1 & 179 & 378 & 0.17 \\
\hline 3 & 114 & 19 & 2 & 110 & 245 & 0.11 \\
\hline 4 & 103 & 32 & 0 & 35 & 170 & 0.07 \\
\hline 5 & 732 & 197 & 4 & 75 & 1009 & 0.45 \\
\hline 6 & 2230 & 465 & 29 & 299 & 3023 & 1.36 \\
\hline 7 & 4974 & 1645 & 224 & 545 & 7388 & 3.32 \\
\hline 8 & 6713 & 5495 & 1041 & 1121 & 14370 & 6.47 \\
\hline 9 & 3988 & 5772 & 1003 & 1780 & 12543 & 5.65 \\
\hline 10 & 1696 & 6886 & 1027 & 2202 & 11811 & 5.32 \\
\hline 11 & 1079 & 8624 & 966 & 2556 & 13225 & 5.95 \\
\hline 12 & 1230 & 9764 & 682 & 3346 & 15022 & 6.76 \\
\hline 13 & 1913 & 10100 & 901 & 3775 & 11689 & 7.51 \\
\hline 14 & 1754 & 9360 & 666 & 3247 & 15027 & 6.76 \\
\hline 15 & 2298 & 9652 & 558 & 3081 & 15589 & 7.02 \\
\hline 16 & 3791 & 9845 & 658 & 3149 & 17443 & 7.85 \\
\hline 17 & 4886 & 9031 & 596 & 3711 & 18224 & 8.20 \\
\hline 18 & 5740 & 7469 & 764 & 4405 & 18378 & 8.27 \\
\hline 19 & 3004 & 5361 & 826 & 4564 & 13755 & 6.19 \\
\hline 20 & 1563 & 3981 & 492 & 3845 & 9881 & 4.45 \\
\hline 21 & 1096 & 2688 & 515 & 2928 & 7227 & 3.25 \\
\hline 22 & 886 & 1560 & 352 & 2134 & 4932 & 2.22 \\
\hline 23 & 846 & 806 & 15 & 1263 & 2930 & 1.32 \\
\hline Total & 51699 & 109424 & 11492 & 49398 & 222012 & 100 \\
\hline
\end{tabular}


assumption that the large load has a large number of EVs, which is acceptable as normally a large load implies many households and thus it implies many EVs. Table 1 shows the number of EVs at each load point [16]. To determine the number of EVs at each time, we used the actual traffic volume from [20]. In [20], the actual number of vehicles at each time is given with different types of purposes such as commutation and business. With the given number of vehicles, it is possible to calculate the traffic ratio at each time. This is simply calculated in (1).

$$
R_{k}=\frac{C_{k}}{C_{t o t}}
$$

where $C_{k}$ refers to the number of vehicles at time $k, C_{t o t}$ refers to the total number of vehicles, and $R_{k}$ refers to the traffic volume ratio at time $k$. Table 2 shows the number of vehicles and traffic volume ratio at each time [16].

By using data from Tables 1 and 2, it is possible to compute the number of EVs at each load and time. Also as average driving period is an hour, the number of charging EVs at time $k$ is the number of EVs at time $k-1[20]$. Calculation is conducted using Eq. (2), and Fig. 2 shows the computed results of L1 and L11, which are the closest and the farthest load from the substation, respectively.

$$
E V_{j . k}=R_{k} \times E V_{j . t o t}
$$

where $E V_{j . k}$ refers to the number of EVs at time $k$ at $j^{\text {th }}$ load. $R_{k}$ refers to the traffic ratio at time $k$. $E V_{j . t o t}$ refers to the total number of EVs at $j^{\text {th }}$ load.

\subsection{Computation on the initial SoC of each EV}

As we set the number of EVs at each load and time, the next step involves computation of the initial SoC of each EV. Estimations of SoC for each EV are one of themost crucial factors for practical analysis of the effect of EVs on the power system. If we were to assume that all of the EVs have an equal initial SoC when they are connected to

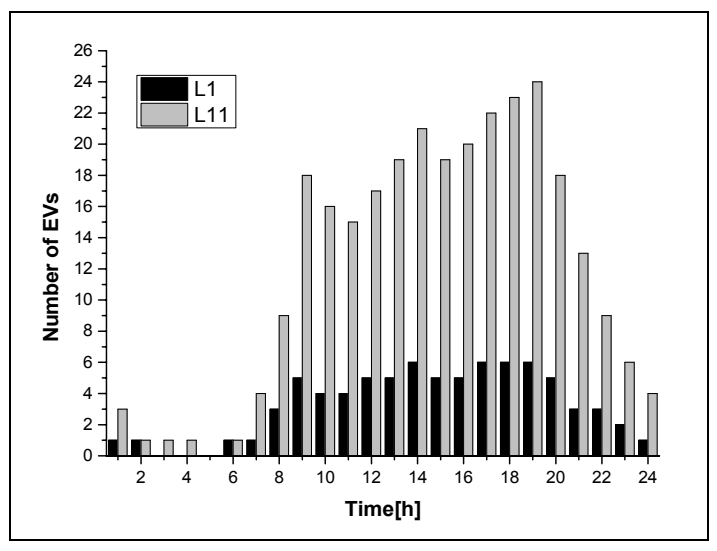

Fig. 2. The number of EVs at each hour (L1, L11)
Table 3. Average daily driving distance depending on the purpose of use

\begin{tabular}{c|c|c|c|c}
\hline & Sample & $\begin{array}{c}\text { Average } \\
(\mathrm{km})\end{array}$ & $\begin{array}{c}\text { Standard } \\
\text { deviation }\end{array}$ & $\begin{array}{c}\text { Range } \\
(\mathrm{km})\end{array}$ \\
\hline Government & 28,354 & 31.2 & 21.5 & 199.8 \\
\hline Private & $4,746,297$ & 38.8 & 21.9 & 384.2 \\
\hline Commercial & 400,416 & 157.5 & 97.8 & 777.0 \\
\hline
\end{tabular}

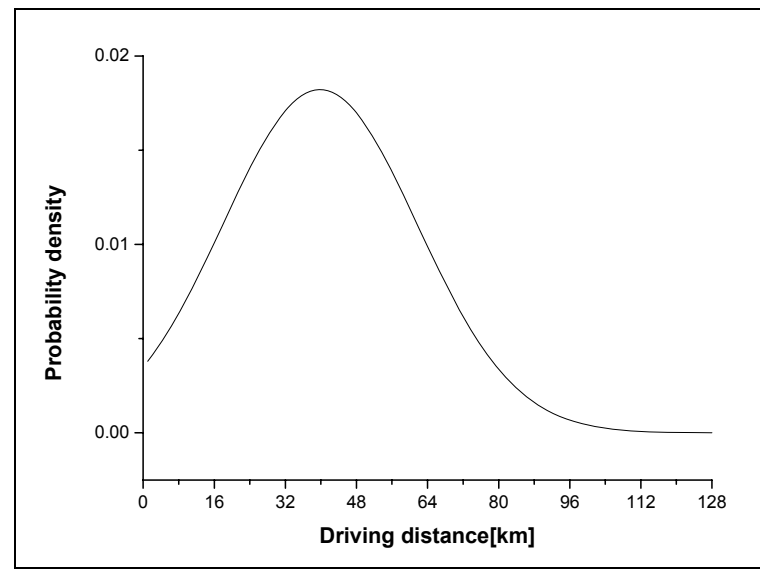

Fig. 3. Gaussian distribution for driving distance

the power system, severe inaccuracy would result in the analysis. Therefore, we used the actual data of the driving pattern for the driving distance and stochastic concept. In terms of a stochastic approach, we used Gaussian distribution. For Gaussian distribution, two variables are required: average and standard deviation of driving distance. Table 3 shows these two variables [21]. In Table 3, we only consider a personal purpose vehicle because it comprises the largest portion of vehicle types. Thus, the average driving distance and standard deviation are set as $38.8 \mathrm{~km}$ and 21.9, respectively.

Using above data, we can calculate the Gaussian distribution of the driving distance with (3) [22].

$$
p(\mu, \sigma)=\frac{1}{\sqrt{2 \pi} \sigma} e^{-\frac{(X-\mu)^{2}}{2 \sigma^{2}}}
$$

where, $\mu$ refers to the average driving distance, $\sigma$ refers to the standard deviation and $X$ is actual driving distance. The calculation results are shown in Fig. 3. If it is assumed that $\mathrm{SoC}$ is linearly proportional to the driving distance, the estimation of SoC for each $\mathrm{EV}$ can be derived from (4) [22].

$$
S o C_{i n i}=\left(1-\frac{\alpha X}{d_{R}}\right) \times 100 \%
$$

where, $S o C_{i n i}$ refers to the initial SoC of each $\mathrm{EV}, d_{R}$ is the maximum driving distance for one charging, $\alpha$ refers to the charging cycle, and $X$ is driving distance. As we assumed 


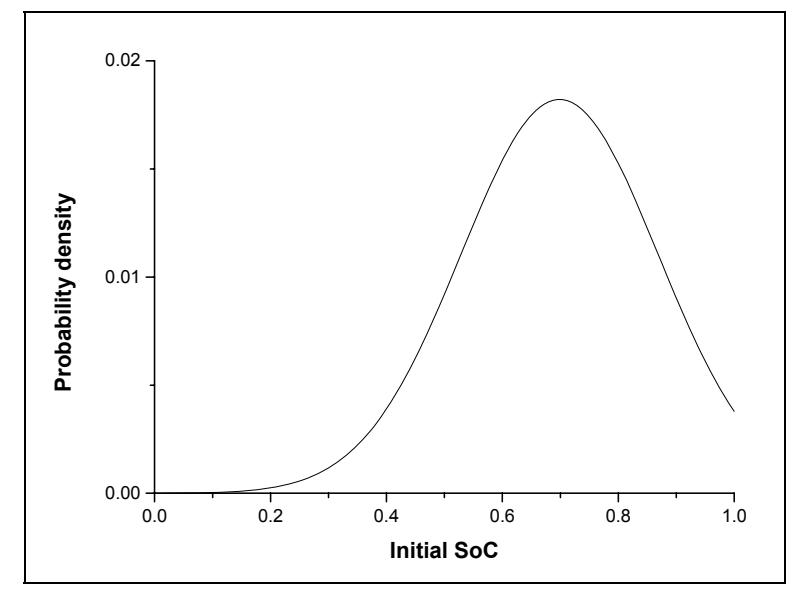

Fig. 4. Gaussian distribution for initial SoC

that $\mathrm{SoC}$ has a linear relationship with driving distance, it is possible to estimate the Gaussian distribution for the initial SoC using (3) and (4). The calculated results are shown in Fig. 4.

The main purpose of the estimation of the initial $\mathrm{SoC}$ at each $\mathrm{EV}$ is to consider the driving pattern of each $\mathrm{EV}$ owner. Depending on the driving distance, the initial SoCs will have different values and this eventually changes the EV loads. Therefore, if the concept of stochastic driving distance is not taken into account, the practical analysis of the effects of EVs cannot be accomplished.

\section{EVs Charging Scheme}

This section introduces the various EV charging schemes including the smart charging scheme. The characteristics of each EV charging scheme are explained in the following subsections.

\subsection{Dumb charging scheme}

As its name implies, the dumb charging scheme does not provide any control scheme. Thus, it is also called uncontrolled charging. In this charging scheme, the EV owners will charge their EV without considering the grid services or electricity price. The main drawback of this charging scheme is the increase of demand power during the peak load period. Typically, EV charging will be conducted when the EV owner returns home from work, which is normally during the peak load period.

\subsection{Off-peak charging scheme}

The off-peak charging scheme was introduced to mitigate the increase in the demand for power for EV charging during the peak load period. It limits EVs to be charged during the peak load period. However, its disadvantage is that although it can prevent the increase of demand power
Table 4. Electricity price for EV charging by KEPCO

\begin{tabular}{c|c|c|c}
\hline \multicolumn{4}{c}{ Electrical charging price for EV (won/kWh) } \\
\hline Time & $\begin{array}{c}\text { Summer } \\
(7 \sim 8)\end{array}$ & $\begin{array}{c}\text { Spring/Autumn } \\
(3 \sim 6 / 9 \sim 10)\end{array}$ & $\begin{array}{c}\text { Winter } \\
(11 \sim 2)\end{array}$ \\
\hline Off-peak & 55.80 & 56.90 & 78.20 \\
\hline Mid-peak & 140.80 & 68.30 & 124.20 \\
\hline Peak & 225.30 & 73.10 & 184.90 \\
\hline
\end{tabular}

during the peak load period, it could introduce another peak load period on the power system. To be specific, if a large number of EV owners charge their vehicle as soon as the peak load period ends, another severe increase of demand power could occur at the beginning of the nonpeak period.

\subsection{Time-of-Use price based charging scheme}

In this charging scheme, the electricity price for EV charging is set differently depending on the time. In the peak load period, the electrical charging price is set as the most expensive price. KEPCO, which is the major power utility in the Republic of Korea, has set the ToU price as shown in Table 4 [23].

According to [24], a $100 \%$ price change may produce around $20 \%$ change in demand. The following assumptions are made considering the tendency and ToU price indicated in Table 4.

1) In peak load period: $80 \%$ of customers who want to charge their EVs during the peak load period will shift their charging time to the off-peak load period.

2) In mid-peak load period: $50 \%$ of customers who want to charge their EVs during the mid-peak load period will shift their charging time to the off-peak load period.

3) In off-peak load period: No customers who want to charge their EVs in an off-peak load period will move their charging time to the mid-peak load or peak load period.

Using the above assumptions, we can estimate the number of charging EVs at each hour. At the same time, we can determine the number of $\mathrm{EV}$ drivers wanting to change their charging time. However, as the ToU price based EV charging scheme merely encourages EV owners to charge their EVs during off-peak load duration, this could lower the load leveling because there is no control for the distribution of the EVs during off-peak load duration.

\subsection{Proposed smart charging scheme}

The previously mentioned charging schemes such as dumb, off-peak, and ToU price based, have drawbacks in terms of the increase of demand power and load factor. The dumb charging scheme can increase the demand power during the peak load period, and during off-peak the charging scheme demand power can even severely increase 
when the peak load duration ends. The ToU price based charging scheme can increase the load factor as there is no control for the distribution of EVs during the off-peak period. In this paper, a smart charging scheme is therefore proposed to prevent the rapid increase of demand power and improve the load factor by controlling the charging of EVs during the off-peak period. The proposed smart charging operates based on the following steps.

1) Measure demand power at each load: As the proposed smart charging scheme uses demand power for controlling the use of power, the measuring demand power at each load should be conducted as the first priority.

$$
P_{k}=V_{k} I_{k}
$$

where $P_{k}$ refers to the demand power on $k$ load and $V_{k}$ and $I_{k}$ refer to the voltage and current on $k$ load, respectively.

2) Judge charging period: The proposed scheme uses the ToU price based charging scheme during the peak and mid-peak load period. That is, the smart charging scheme operates only during the off-peak load period. Thus, a classification of the charging period is required.

$$
\left\{\begin{array}{l}
S_{k}=1(\text { Off }- \text { peakload period }) \\
S_{k}=0(\text { Mid }- \text { peak and peak load period })
\end{array}\right.
$$

where, $S_{k}$ refers to the control signal of smart charging scheme. If $S_{k}$ is equal to 1 , the proposed scheme operates on its own. On the other hand, when $S_{k}$ is equal to 0 , the proposed scheme uses the ToU price based EV charging scheme.

3) Estimation of the state of charging EVs at each hour: The number of charging EVs at each hour needs to be estimated in order to distribute the charging EVs during the off-peak load period. For this purpose, the matrix concept is used in this paper. To estimate the state of charging EVs, this paper assumed two conditions:

(1) All EVs are charged with constant current

(2) All the EVs would keep charging by the time SoC reaches $100 \%$.

Using Gaussian distribution for SoC and the two assumptions given above, the required charging time can be estimated for each EV. The estimated data are stored in the matrix.

$$
\begin{gathered}
\boldsymbol{A}_{\text {chage }}=\sum_{n=1}^{24} A_{n}, \boldsymbol{A}_{\boldsymbol{n}}=\left[\begin{array}{ccc}
a_{(1,1)}^{n} & \cdots & a_{(1,24)}^{n} \\
\vdots & \ddots & \vdots \\
a_{\left(r_{n}, 1\right)}^{n} & \cdots & a_{\left(r_{n}, 24\right)}^{n}
\end{array}\right] \\
\left\{\begin{array}{c}
a_{(x, y)}^{n}=1\left(n \leq y \leq T_{\text {n.req } x}+n\right) \\
a_{(x, y)}^{n}=0\left(y \geq T_{n \text { req } x}+n \text { or } y<n\right)
\end{array}\right.
\end{gathered}
$$

where, $\boldsymbol{A}_{\text {chage }}$ is the matrix showing charging states of all EVs in a specific region. $\boldsymbol{A}_{\boldsymbol{n}}$ indicates charging state of all EVs which started charging at $\mathrm{n}$ and $a_{(x, y)}^{n}$ indicates the charging state of the $x^{\text {th }} \mathrm{EV}$, which started charging from $\mathrm{n}$, at time $\mathrm{y} . T_{\text {n.req } x}$ indicates the required charging time for the $x^{\text {th }} \mathrm{EV}$, which started charging from $\mathrm{n}$, and $n$ indicates the time which has the value between 1 to $24 . r^{n}$ indicates the total number of vehicle which start to charge at time n. When $a_{(x, y)}^{n}$ has a value 1 , then the $x^{\text {th }} \mathrm{EV}$ will be charged at time $y$. The total number of EVs which will charged at time $n$ can be calculated using (8).

$$
\begin{aligned}
N_{E V . n}= & \left(A_{\operatorname{charge}(n, 1)}+A_{\operatorname{charge}(n, 2)}+\cdots\right. \\
& \left.+A_{\operatorname{charge}\left(n, r_{\text {cum }}^{n}-1\right)}+A_{\operatorname{charge}\left(n, r_{\text {cum }}^{n}\right)}\right)
\end{aligned}
$$

where, $r_{\text {cum }}^{n}$ indicates the total cumulative number of charging EVs at time $n$.

4) Estimation of required number of EVs for load leveling: The above section explains the estimation of the total charging EVs at each hour. We can now predict the total demand power of the power system. However, the total number of charging EVs calculated in the above subsection does not include the EVs in which the charging time was changed due to the ToU price based charging scheme. Thus, the next step is the calculation of the

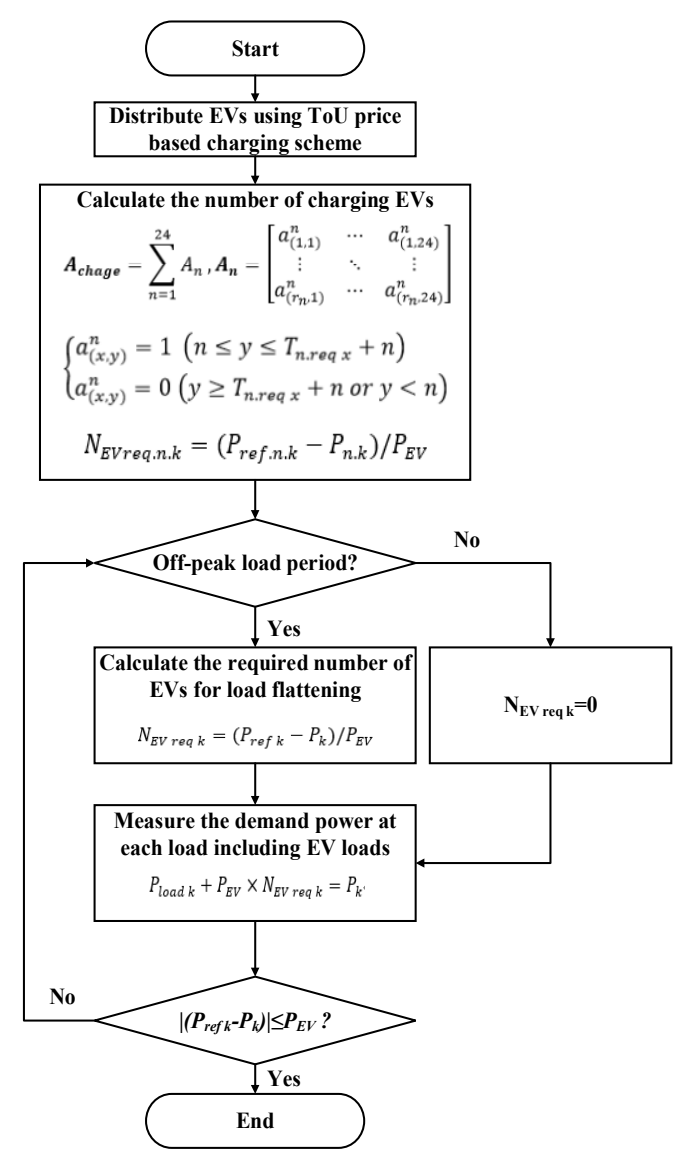

Fig. 5. Smart EV charging scheme control algorithm 
required number of EVs for load leveling using the shifted EVs. The required number of EVs can easily be found using (9).

$$
N_{\text {EVreq.n.k }}=\left(P_{\text {ref.n.k }}-P_{n . k}\right) / P_{E V}
$$

where $N_{\text {EVreq.n.k }}$ refers to the required number of EVs for load leveling at time $\mathrm{n}$ on $k$ load. $P_{\text {ref.n.k }}$ refers to the reference demand power at time $\mathrm{n}$ on $k$ load and $P_{n . k}$ refers to the system demand power at time n on $k$ load and $P_{E V}$ refers to the power consumption of a single EV.

5) Feedback Loop: The main purpose of feedback loop is to prepare unexpected change of power system. The proposed algorithm measures demand power and set the number of charging EVs. If there is any unexpected change in power system after set the number of charging EVs, however, it may not be the optimal numbers. Using feedback loop, it can adjust optimal number of charging EVs even with the unexpected changes in power system.

All the procedures mentioned above are summarized in Fig. 5.

\section{Case Studies}

As mentioned previously, for practical analysis, we performed case studies on the actual distribution system of KEPCO with actual traffic volume data. Additionally, as the Republic of Korea has different load patterns and traffic patterns, we focus on the data in summer that has frequent variations in the demand power and a considerable gap between minimum demand power and peak demand power in order to analyze the worst case.

\subsection{Test distribution system}

Fig. 6 shows a single line diagram of the distribution system. As explained in section II, we chose the bottom portion of the distribution system (shown in Fig. 6), which is a specific region in Seoul, Republic of Korea.

The main reason for considering only the bottom part of the distribution system is to increase the accuracy of the analysis. The upper portion of the system has an interconnection area that causes uncertainties and complexities in the analysis [17]. To be more specific, if there is no interconnection area, we can only consider one specific area and can use the data of that area. However, if there is an interconnection area, we can adopt neither the data of one specific area nor the combined data of several areas. In that case, we need to consider various conditions such as traffic flow and density of population. In this paper, we therefore exclude the possibilities of complexities and uncertainties by focusing only on the bottom portion of the system.

\subsection{Electric vehicles}

An EV consists of several components such as an electrical motor, a battery, an on-board charger, etc. However, since in most EVs the ignition is turned off during charging, we only consider the battery and the charger. Also, as most on-board chargers are designed to have a 2 to $5 \mathrm{~kW}$ rating considering the power contract is for family use, we assumed that the charger has a $3.3 \mathrm{kWh}$ charging rate [25]. The battery itself is set to have a $16.5 \mathrm{kWh}$ capacity [25]. That is, an EV with a $0 \mathrm{SoC}$ would take 5 hours to completely charge when connected to the power system.

\subsection{Dumb EV charging scheme}

As the dumb EV charging scheme does not provide any control scheme, most EVs are concentrated on a peak load period. Fig. 7 shows a comparison of the demand power on the system in a dumb EV charging scheme and base case

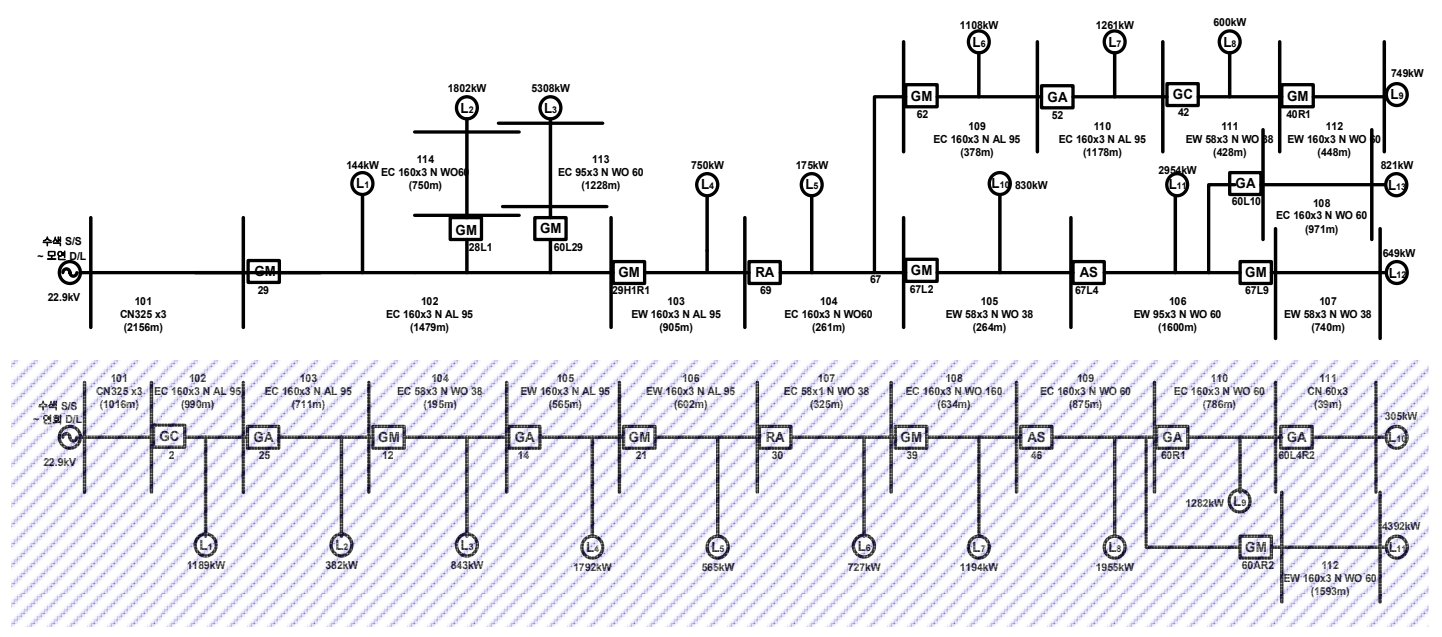

Fig. 6. Single line diagram of used distribution system 


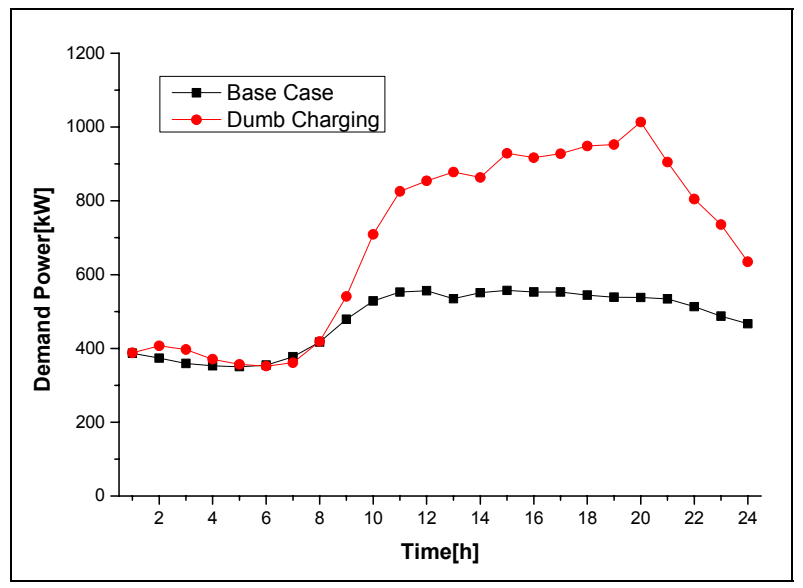

Fig. 7. The demand power of the system (Base case vs. dumb charging scheme)

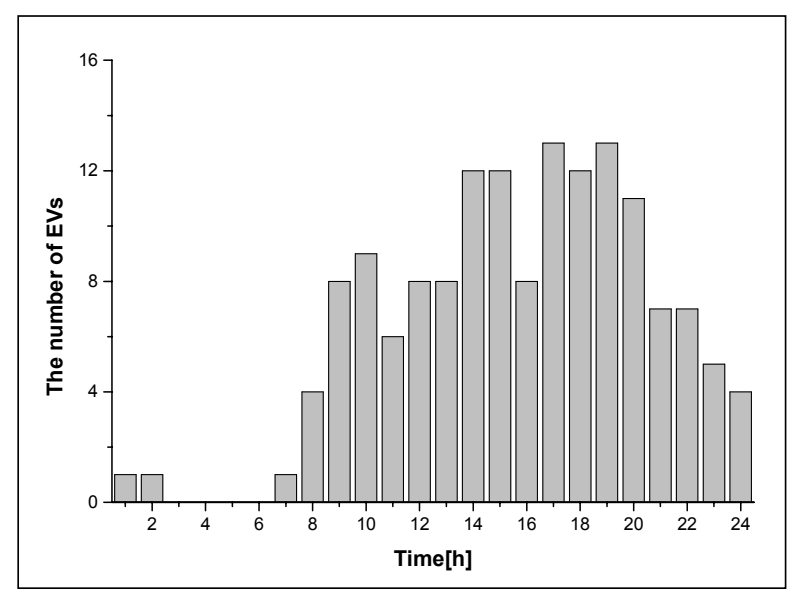

Fig. 8. The number of charging EVs at each hour (Dumb charging, L1)

without EVs. Also, Fig. 8 shows the number of EVs at each hour under a dumb charging scheme. As shown in Fig. 7, the peak demand power for dumb charging reaches 1,013 $\mathrm{kW}$, which is 1.82 times larger than that of the base case. Moreover, it is shown that the load factor would decrease with the dumb EV charging scheme. The load factor is derived from the ratio of the average demand power to peak demand power. For the base case, the load factor is $85.71 \%$. However, when the dumb EV charging scheme is adopted, the load factor decreases to $67.80 \%$. As a low load factor indicates the need for additional power sources and could cause severe voltage variation, the dumb charging scheme should not be used and an additional solution is required to use the charging scheme.

\subsection{ToU price based EV charging scheme}

When the ToU price based EV charging scheme is used, it could limit the increased power demand during the peak load period. However, as explained above, the ToU price

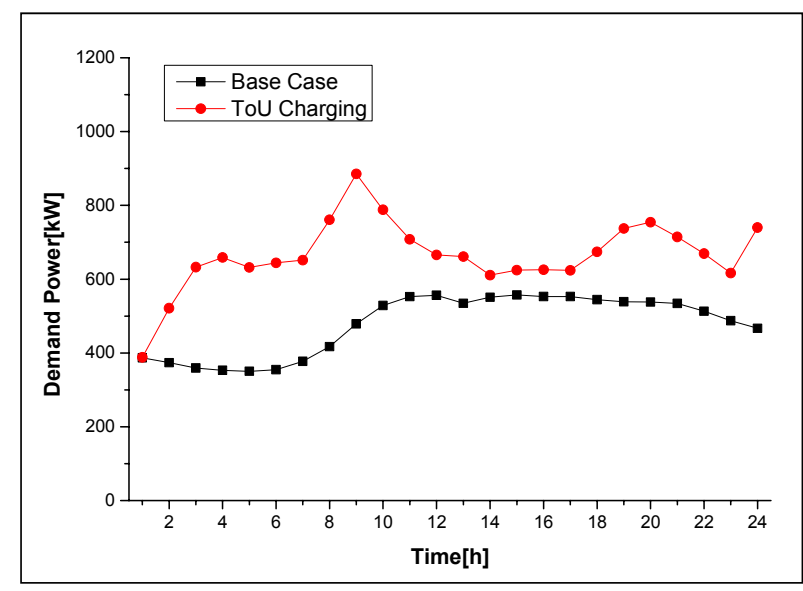

Fig. 9. The demand power of the system (Base case vs. ToU charging scheme)

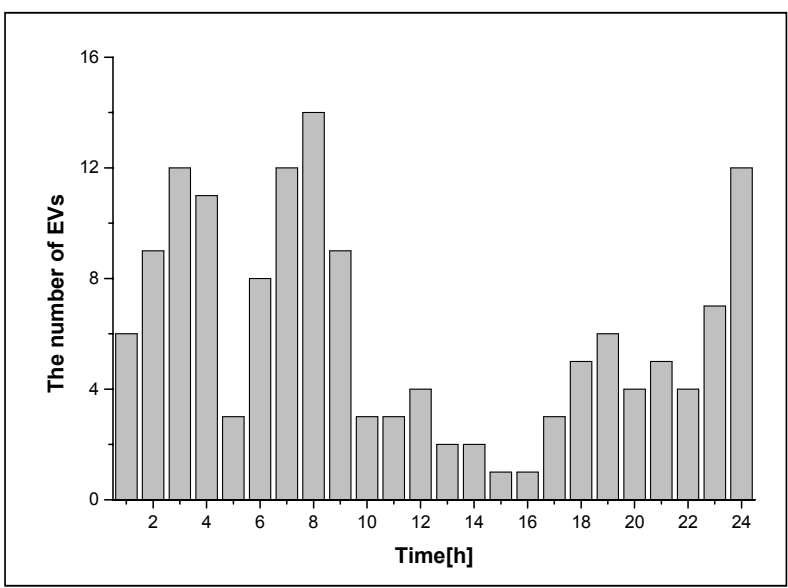

Fig. 10. The number of charging EVs at each hour (ToU price based charging, L1)

based EV charging scheme only encourages EV owners to charge their EV during the off-peak load period. In other words, there is no additional control scheme, once the charging of the EVs commences during off-peak time. Due to this characteristic, although peak demand power is relatively lower than that of the dumb charging scheme, fluctuation still occurs in the demand power of the system as shown in Fig. 9. Also, in contrast to the dumb charging scheme, most charging EVs are concentrated in the offpeak period in the ToU price based EV charging scheme as shown in Fig. 10.

In the case of the ToU charging scheme, the peak demand power and load factor are $885.2 \mathrm{~kW}$ and $75.25 \%$, respectively. Even after the ToU charging scheme is adopted, the load factor is still considerably lower than that of the base case.

\subsection{Smart charging scheme}

The main difference between the ToU price based EV 


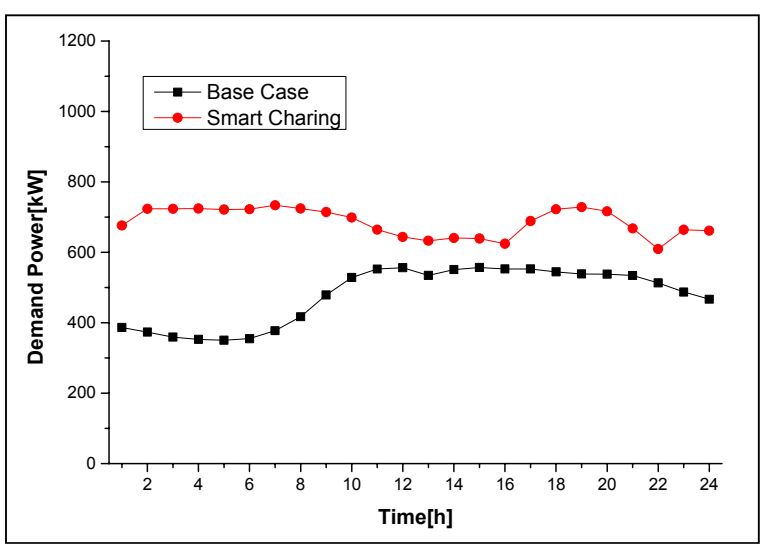

Fig. 11. The demand power of the system (Base case vs. smart charging scheme)

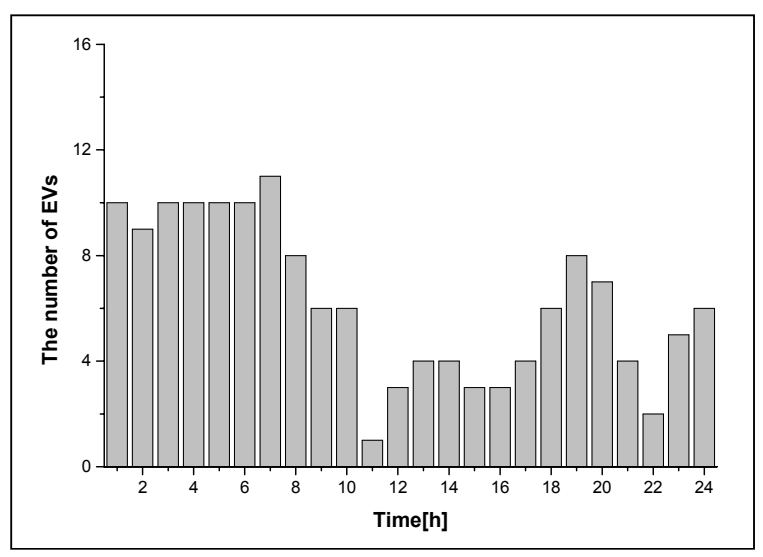

Fig. 12. The number of charging EVs at each hour (Smart charging, L1)

charging scheme and the smart EV charging scheme is the distribution of EVs during the off-peak period. The smart charging scheme determines the number of charging EVs during the off-peak load period, considering the demand power of the system. The demand power of the system and the number of charging EVs at each hour when the smart charging scheme is adopted are shown in Fig. 11 and Fig. 12 , respectively.

When the smart EV charging scheme is adopted, the peak demand power of the system reaches $733.77 \mathrm{~kW}$, which is $208.65 \mathrm{~kW}$ larger than that of the base case. In this case, the load factor increases to $93.5 \%$. Also, the average demand power reaches $686.05 \mathrm{~kW}$, which implies that the system operator can increase the portion of the base power in the system. In terms of economical and system operational aspects, an increase of the portion of the base power could bring considerable benefits. Thus, even though the proposed smart charging scheme increases the demand power of the system compared to the base case, it shows excellent performance. Fig. 13 and Table 5 show simulated results depending on each type of EV charging scheme.
Table 5.Summarization of analysis results

\begin{tabular}{c|c|c|c|c}
\hline & Base case & $\begin{array}{c}\text { Dumb } \\
\text { charging }\end{array}$ & $\begin{array}{c}\text { ToU } \\
\text { charging }\end{array}$ & $\begin{array}{c}\text { Smart } \\
\text { charging }\end{array}$ \\
\hline $\begin{array}{c}\text { Average demand } \\
\text { power(kW) }\end{array}$ & 477.4004 & 686.99 & 666.08 & 686.05 \\
\hline $\begin{array}{c}\text { Peak demand } \\
\text { power(kW) }\end{array}$ & 556.99 & 1013.2 & 885.2 & 733.77 \\
\hline Load Factor(\%) & 85.71077 & 67.80 & 75.25 & 93.50 \\
\hline
\end{tabular}

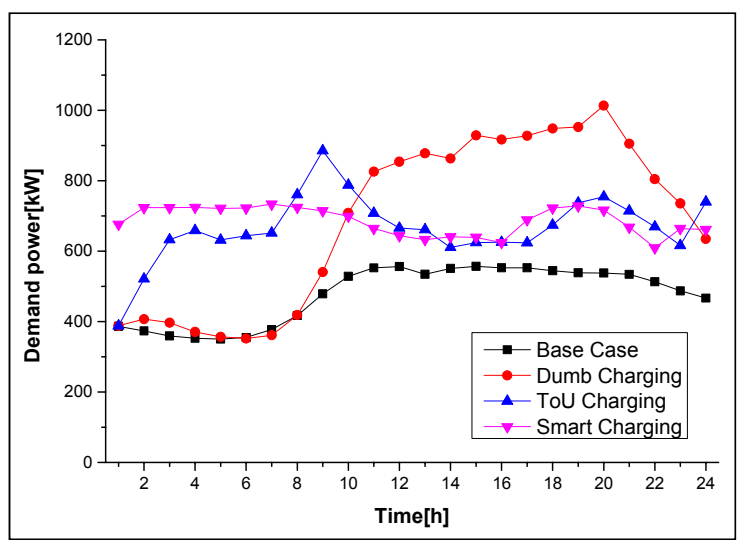

Fig. 13. The demand power of the system for each types of charging schemes

\section{Conclusion}

With the increased interest in EVs, the need for the analysis of EVs also increases. Without a thorough analysis of the possible effects of EVs on power system, it would be impossible to commercialize EVs. In terms of the power system, the charging of EVs could cause various adverse effects such as voltage variations, frequency variations, decrease of load factor, increase of demand power, and decrease of load factor, etc. Among these, we focused on the demand power and load factor of the system. Several types of EV charging schemes have previously been proposed. In the case of the dumb EV charging scheme, the demand power could increase during the peak load period, as there is no control scheme. Although an off-peak EV charging scheme was proposed to limit the increase in the demand power during the peak load period, the demand power still causes a problem, as it would rapidly increase as soon as the peak load period ends. The ToU price based EV charging scheme uses different charging prices, depending on the time to limit the charging of EVs in the peak load period and shift it to an off-peak load period. However, as it does not provide a charging scheme except for the ToU price, proper valley filling and load leveling cannot be accomplished. Thus, new EV charging schemes are required.

The proposed smart EV charging scheme can be divided into two main parts. First, it calculates the number of charging EVs at each hour using the ToU price based 
charging scheme. It then determines the distribution of EVs at each hour considering the demand power of the system using the shifted EVs due to the ToU price based charging scheme. In addition, for practical analysis, we not only consider the actual driving pattern of the EV owners and the initial SoC information, but also the use distribution system based on actual data.

Through a case study, it is shown that the proposed smart charging scheme provides excellent performance. Compared to the previous charging schemes, the proposed smart charging scheme shows an improved load factor of $93.5 \%$, which is an even better result than that of the base case. Also, in terms of demand power, the proposed charging scheme limits the rapid increase of demand power and reduces the peak demand power to $733.77 \mathrm{~kW}$, which is $279.23 \mathrm{~kW}$ lower than that of dumb charging and 151.43 $\mathrm{kW}$ lower than that of the ToU price based charging scheme, as shown in Table 5.

The low load factor means that there are considerably large gaps between the average demand power and the peak demand power. In other words, if the load factor of the system decreases due to EVs, additional power sources are required. However, if we can properly schedule the number of charging EVs, a system operator can manage the system without any additional power sources, while resulting in an even better load factor. Therefore, it is expected that the proposed smart EV charging scheme can contribute to stable power system management. Moreover, by eliminating the possible risks, it could accelerate the popularization of EVs.

\section{Acknowledgements}

This work was supported by the National Research Foundation of Korea(NRF) grant funded by the Korea government(MSIP) (No. 2015R1A2A1A10052459).

This work was supported by "Human Resources Program in Energy Technology" of the Korea Institute of Energy Technology Evaluation and Planning (KETEP), granted financial resource from the Ministry of Trade, Industry \& Energy, Republic of Korea. (No. 20164030200980)

\section{References}

[1] "Toward an Ontario action plan for plug-in electric vehicles (PEVs)", WISE Report to Ontario's Centers for Excellence, May. 2010

[2] Jonathan Donadee and Marija Ilić, "Stochastic CoOptimization of Charging and Frequency Regulation by Electric Vehicles", North American Power Symposium (NAPS), 2012

[3] Sekyung Han, Soohee Han, "Economic Feasibility of V2G Frequency Regulation in Consideration of Battery Wear", Energies Vol. 6, pp. 748-765, 2013
[4] Yuki Mitsukuri, Ryoichi Haraa, Hiroyuki Kitaa, Eiji Kamiyab, Shoji Takib and Eiji Kogureb, "Study on Voltage Regulation in a Distribution System Using Electric Vehicles - Optimal Real and Reactive Power Dispatch by Centralized Control", Journal of International Council on Electrical Engineering, Vol. 3, No. 2, pp.134 140, 2013

[5] C. Roe, F. Evangelos, J. Meisel, A. P. Meliopoulos, and T. Overbye, "Power system level impacts of PHEVs," in Proc. 42nd Hawaii International Conference on System Sciences 2009, pp. 1-10

[6] L. Pieltain Fernandez, T. Gomez San Roman, R. Cossent, C. M. Domingo, and P. Frias, "Assessment of the impact of plug-in electric vehicles on distribution networks" IEEE Transaction on Power System, vol. 26, no. 1, pp. 206-213. Feb. 2011

[7] K. Dyke, N. Schofield, and M. Barnes, "The impact of transport electrification on electrical networks" IEEE Transaction on Industrial Electronics, vol. 57, no. 12 , pp. $3917-3926$, Dec. 2010

[8] J. Taylor, A. Maitra, M. Alexander, D. Brooks, and M. Duvall, "Evaluation of the impact of plug-in electric vehicle loading on distribution system operations," IEEE Power Energy Society General Meeting, 2009

[9] A. Boulanger, A. Chu, S. Maxx, D. Waltz, "Vehicle electrification: Status and issues", Proceedings of IEEE, vol. 99, no. 6, pp.1116-1138, Jun. 2011

[10] O. Sundstorm, C. Binding, "Flexible charging optimization for electric vehicles considering distribution grid constraints", IEEE Transaction on Smart Grid, vol. 3, no. 1, pp. 26-37, Mar. 2012

[11] K. Clement-Nyns, E, Haesen, and J. Driesen, "The impact of charging plug-in hybrid electric vehicles on residential distribution grid", IEEE Transaction on Power System, vol. 25 no. 1, pp.371-380, Feb. 2010

[12] E. Sortomme, M. Hindi, S. MacPherson, and S. Venkata, "Coordinated charging of plug-in hybrid electric vehicles to minimize distribution system losses", IEEE Transaction on Smart Grid, vol. 2, no. 1, pp. 198-205, Mar. 2011

[13] K. P. Schneider, C. E. Gerkensmeyer, M. C. W. Kintner-Meyer, R. Fletcher, "Impact assessment of plug-in hybrid vehicles on pacific northwest distribution systems" Power Energy Society General Meeting Jul. 2008

[14] L. Gan, U. Topcu, S. Low, "Optimal decentralized protocol for electric vehicle charging", IEEE Transaction on Power System, vol. 28, no, 2, pp. 940951, May. 2013

[15] Z. Ma, D. s. Callaway, I. A. Hiskens, "Decentralized charging control of large populations of plug-in electric vehicles", IEEE Conference of Decision and Control, 2010

[16] Keon-Woo Park, Hun-Chul Seo, Chul-Hwan Kim, Chang-soo Jung, Yeon-Pyo Yoo, Yong-Hoon Lim, "Analysis of the Neutral Current for Two-Step- 
TypePoles in Distribution Lines," IEEE Transactions onPower Delivery, Vol. 24, pp. 1483-1489, 2009

[17] Soon-Jeong Lee, Jun-Hyeok Kim, Doo-Ung Kim, Hyo-Sang Go, Chul-Hwan Kim, Eung-Sang Kim and Seul-Ki Kim, "Evaluation of Voltage Sag and Unbalance due to the System connection of Electric Vehicles on Distribution Sytem", JEET, Vol. 9, No. 2, pp. 452-460, 2014

[18] Statics Korea, http://www.index.go.kr

[19] Korea Smart Grid Institute, "A Research of Charging Infrastructure for Electric Vehicle," 2010

[20] Korea Transport Database, http://www.ktdb.go.kr

[21] Dong-Su Kang and Ju-Heu Kim, "Investigation of actual state of driving distance of vehicle in 2008", Korea Transportation Safety Authority

[22] Kejun Qian, Chengke Zhou, Malcolm Allan, Yue Yuan, "Modeling of Load Demand Due to EV Battery Charging in Distribution Systems", IEEE Transaction on Power Systems, vol. 26, no. 2, May 2011

[23] Korea Electric Power Corporation, "Electric Price", 2013

[24] EPRI, Electric Electricity Research Institute, January, "Price Elasticity of Demand for Electricity: A Primer and Synthesis", 2008

[25] I .H. Park, C. Y. Won, C. H. Kim, W. S. Na, B. K. Lee, "Understanding of electrical power technology for green car”, HANTEEMEDIA, 2011

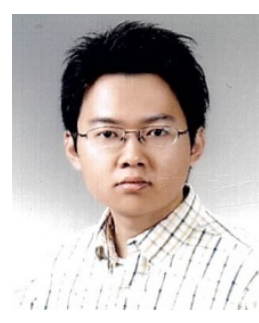

Jun-Hyeok Kim He received B.S. and M.S. degrees in Electrical, Electronic and Computer Engineering from Sungkyunkwan University, 2011 and 2013, respectively. At present, he is working on his Ph.D. course in Electrical, Electronic and Computer Engineering from Sungkyunkwan University. His research interests include power system transients, protection and stability with electric vehicle.

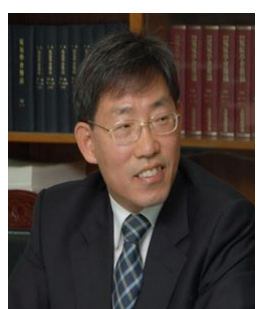

Chul-Hwan Kim He received his B.S. and M.S. degrees in Electrical Engineering from Sungkyunkwan University, South Korea, 1982 and 1984, respecttively. He received a Ph.D. degree in Electrical Engineering from Sungkyunkwan University in 1990. In 1990 he joined Cheju National University, Cheju, South Korea, as a full-time Lecturer. He has been a visiting academic at the University of BATH, UK, in 1996, 1998, and 1999. Since March 1992, he has been a professor in the College of Information and Communication, Sungkyunkwan University, South Korea. His research interests include power system protection, artificial intelligence application for protection and control, the modelling / protection of underground cable and EMTP software. 